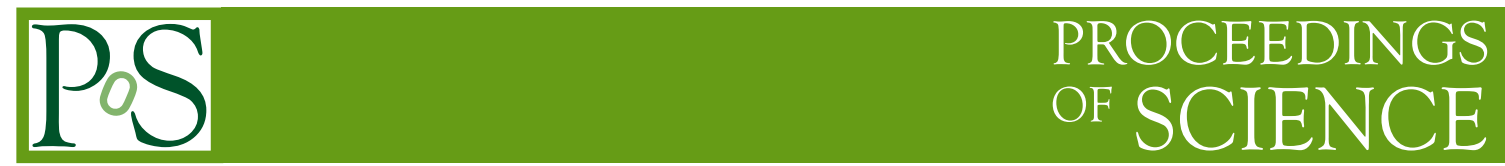

\title{
Search for Long-lived particles with the ATLAS detector
}

\author{
Masahiko Saito* \\ On behalf of the ATLAS Collaboration \\ The University of Tokyo (JP) \\ E-mail: masahiko.saitodcern.ch
}

Several supersymmetric models predict the production of meta-stable supersymmetric particles. Such particles, if charged, may be detected through the disappearing tracks. The poster presents recent results from the disappearing track analysis based on an integrated luminosity of $36.1 \mathrm{fb}^{-1}$ of $p p$ collisions at a centre of mass energy of $13 \mathrm{TeV}$ with the ATLAS detector at the LHC.

The European Physical Society Conference on High Energy Physics

5-12 July

Venice, Italy

${ }^{*}$ Speaker. 


\section{Introduction and motivation}

Supersymmetry theory is one of the most powerful theories beyond the standard model. It predicts a set of new particles; each of them is a supersymmetric partner of a standard model particle. The lightest supersymmetric particle (LSP) is a good candidate for dark matter. Especially when a pure wino, which is a supersymmetric partner of the weak boson field, is the LSP, the chargino mass is highly degenerate with the neutralino mass because winos form a triplet. Therefore a chargino becomes long-lived enough to reach an inner detector of a high-energy collider experiment. If the mass difference between a chargino and a neutralino is about $160 \mathrm{MeV}$ as in the case of wino LSP, a chargino decays into a neutralino, which cannot be detected due to zero electric charge or strong charge, and a pion whose momentum is so low that it cannot be reconstructed as a track. Therefore a long-lived wino produced in the ATLAS detector [1] at the LHC will be observed with the disappearing track signature (Figure 1). In the pure wino case, the chargino lifetime is 0.2 nanosecond, corresponding to $\mathrm{O}(10) \mathrm{cm}$ that is the same scale with the pixel detector in the ATLAS inner detector. These short disappearing tracks are reconstructed by a new special tracking algorithm, which can be used thanks to a new inner pixel layer $[2,3]$.

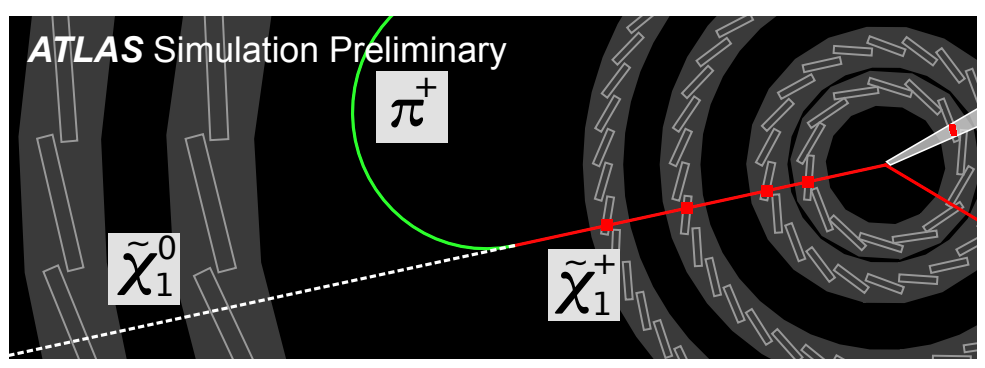

Figure 1: Illustration of a charged wino production event [4]. The chargino (red line) decays into the pion (green) and the neutralino (dotted line).

\section{Analysis overview}

To achieve complementary searches, two signal production processes are considered, electroweakgaugino pair production (EW channel) and gluino pair production (strong channel). Two different kinematic selections are prepared for each production to maximize the sensitivity. For EW channel, at least one initial state radiation jet is required to trigger events. For strong channel, at least three jets are required because signal events are expected to have multi-jets from gluino cascade decays. High missing transverse momentum is required in both channels because long-lived charginos that become short disappearing tracks don't leave an energy deposit in the calorimeter. After the kinematic selection, at least one disappearing track candidate is required.

\section{Tracklet Reconstruction}

The ATLAS inner detector consists of three components: a pixel detector, a strip semiconductor tracker (SCT), and a transition radiation tracker. The pixel detector, which is the innermost 
detector, spans the radius range from $3 \mathrm{~cm}$ to $12 \mathrm{~cm}$ from the beam interaction point. The insertable $B$-layer, which is the innermost layer in pixel detector, was installed at $3 \mathrm{~cm}$ before Run 2 .

The ATLAS standard tracking algorithm requires at least 7 hits in the silicon detector (pixel and SCT) per track to reduce fake tracks. However, this cannot reconstruct target signals because disappearing tracks from the expected signals decay at shorter radii and don't have more than 7 silicon hits. To recover a signal acceptance, the new tracking algorithm was developed. The tracks reconstructed by the new tracking algorithm are called pixel-tracklets. The pixel-tracklets are reconstructed by using at least 4 hits in the pixel detector and no SCT hits on tracks are required. The pixel-tracklet reconstruction recovers the acceptance for signals decaying between the pixel detector and the SCT detector. Figure 2 shows the signal reconstruction efficiency. Green points show the reconstruction efficiency of the standard tracks, which is reconstructed by the standard tracking algorithm, and red points show that of the pixel-tracklets. The pixel-tracklets reconstruction can increase the signal acceptance for 0.2 nanosecond chargino by a factor of 10 .

The transverse momentum $\left(p_{\mathrm{T}}\right)$ resolution of tracklets is worse than standard tracks because their track length is short. Pixel-tracklet $p_{\mathrm{T}}$ resolution was measured in data and used in the background estimation and the signal track $p_{\mathrm{T}}$ modelling.

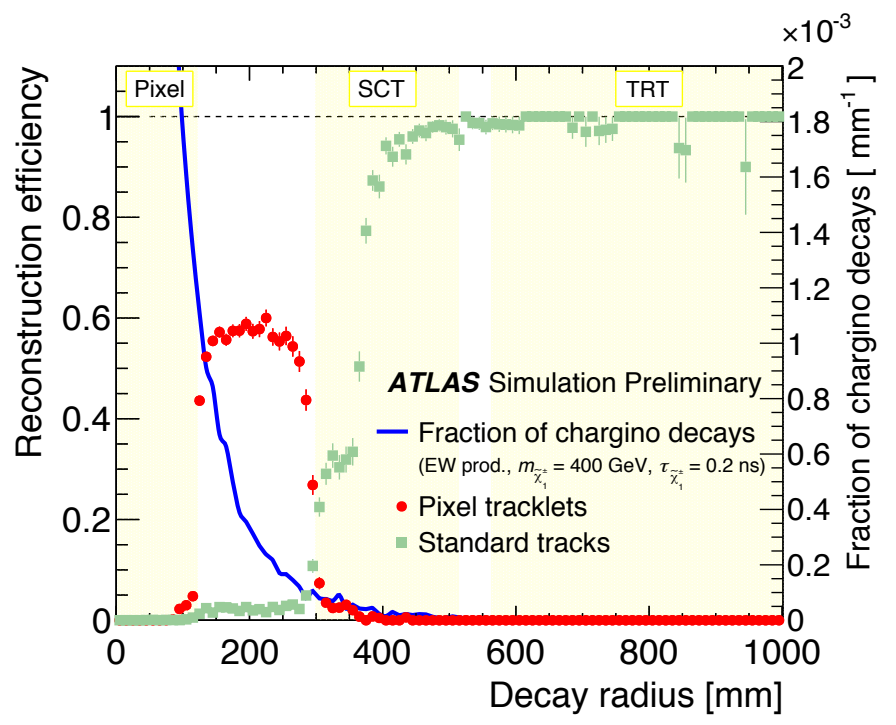

Figure 2: The signal reconstruction efficiency as a function of decay radius [4]. Red dots show the pixeltracklet reconstruction efficiency of the new tracking algorithm, while green dots show that in the standard tracking algorithm. A blue line shows the expected chargino decay radius distribution.

\section{Background estimation and signal extraction}

Two background components are considered. The first component is interacting standard model particles, which are charged particles interacting with materials in the detector and failing to be reconstructed as standard tracks. The second is fake-tracklet background, which comes from unphysical tracks reconstructed from wrong combinations of pixel clusters. 
To discriminate signal from background, an unbinned likelihood fit is performed on the pixeltracklet $p_{\mathrm{T}}$ distribution. The signal track $p_{\mathrm{T}}$ template is estimated from Monte Carlo simulation and smeared by the pixel-tracklet $p_{\mathrm{T}}$ resolution measured from data. Interacting background $p_{\mathrm{T}}$ templates are estimated from background pure regions (control regions) and smeared to estimate $p_{\mathrm{T}}$ shapes in the signal region. The fake-tracklet background $p_{\mathrm{T}}$ template is estimated from tracks with a large transverse impact-parameter, which are dominated by fake-tracklets.

\section{Result and interpretation}

Figure 3 shows the results of the pixel-tracklet $p_{\mathrm{T}}$ fit. No excess is found in both channels. Figure 4 shows exclusion limits. For a pure wino whose lifetime is 0.2 nanosecond, charginos with a mass up to $430 \mathrm{GeV}$ are excluded at $95 \%$ confidence level (CL). For a light gluino case, gluinos with a mass up to $1.6 \mathrm{TeV}$ are excluded at $95 \% \mathrm{CL}$ assuming chargino mass of $430 \mathrm{GeV}$. For a gluino-wino mass compressed case, chargino with a mass up to $1.05 \mathrm{TeV}$ are excluded at 95\% CL.

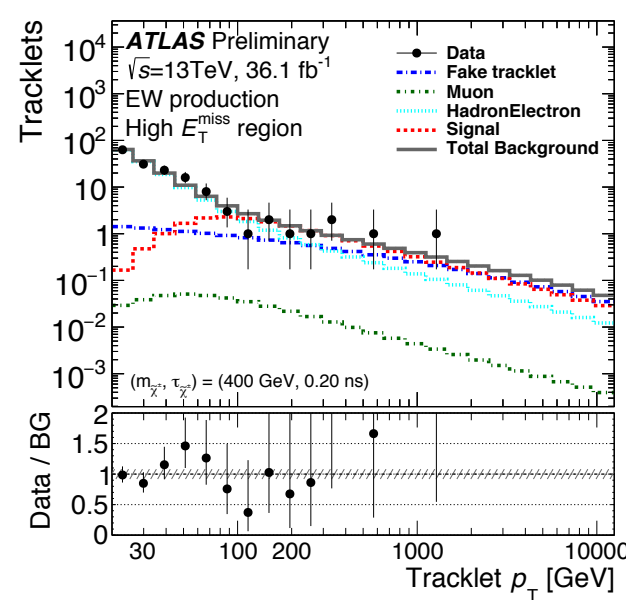

(a)

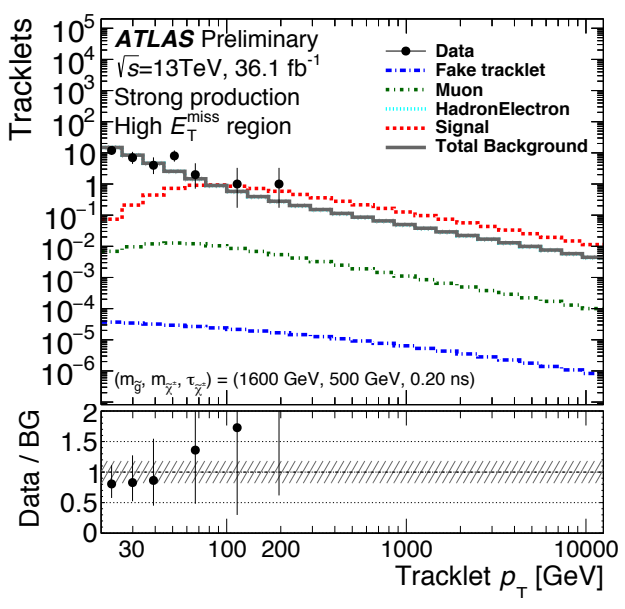

(b)

Figure 3: Results of pixel-tracklet $p_{\mathrm{T}}$ fit in two channels [4]: (a) electroweak channel, and (b) strong channel. Black dots show observed data. Gray lines show total expected backgrounds. Red lines show the expected signal $p_{\mathrm{T}}$ distribution.

\section{Conclusion}

Recent results of the disappearing track analysis based on an integrated luminosity of 36.1 $\mathrm{fb}^{-1}$ in 2015 and $2016 p p$ collisions data at $\sqrt{s}=13 \mathrm{TeV}$ with the ATLAS detector at the LHC are reported. The new special tracking algorithm was developed to recover the efficiency for short tracks, which became possible thanks to the new pixel layer. This increases the signal acceptance for the pure wino by a factor of 10 . No excess is found in this search and tightest limits are set on the pure wino LSP mass. 


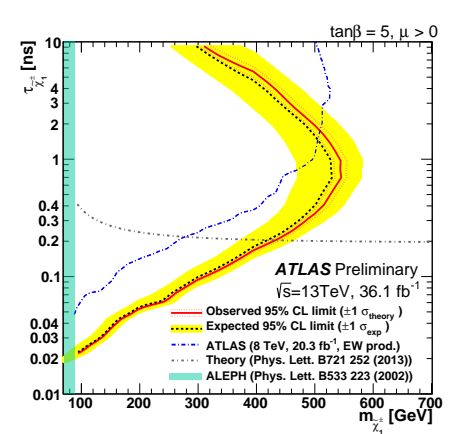

(a)

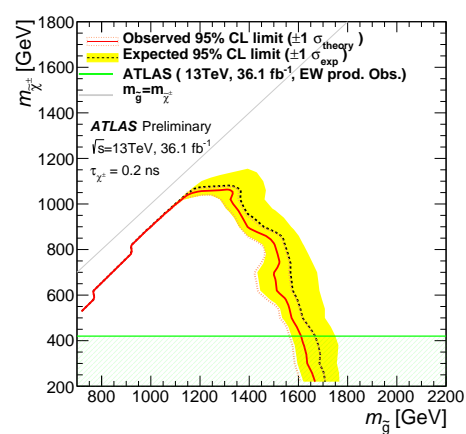

(b)

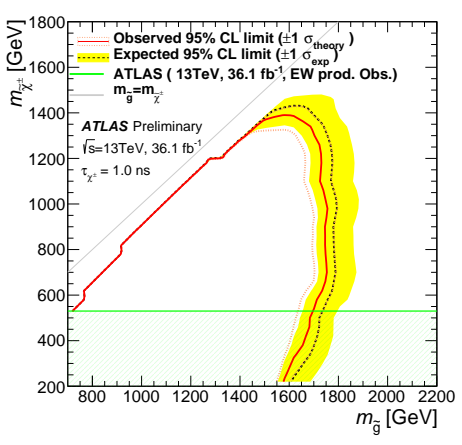

(c)

Figure 4: Exclusion limits at 95\% CL in two channels [4]: (a) electroweak channel, and strong channel with a chargino lifetime of (b) 0.2 nanosecond and (c) 1.0 nanosecond. The red line shows the observed limit. The black dotted line in Figure (a) shows a prediction of a lifetime of chargino in the pure wino LSP case.

\section{References}

[1] ATLAS Collaboration, The ATLAS Experiment at the CERN Large Hadron Collider, JINST 3 (2008) S08003.

[2] ATLAS Collaboration, ATLAS Insertable B-Layer Technical Design Report, ATLAS-TDR-19, https://cds.cern.ch/record/1291633

[3] ATLAS Collaboration, ATLAS Insertable B-Layer Technical Design Report Addendum, ATLAS-TDR-19-ADD-1, https://cds. cern.ch/record/1451888

[4] ATLAS Collaboration, Search for long-lived charginos based on a disappearing-track signature in pp collisions at $\sqrt{s}=13 \mathrm{TeV}$ with the ATLAS detector, ATLAS-CONF-2017-017, https://cds.cern.ch/record/2258131 